Case Report

\title{
Mucoepidermoid Carcinoma Associated with Osteosarcoma in a True Malignant Mixed Tumor of the Submandibular Region
}

\author{
Dario Marcotullio, ${ }^{1}$ Marco de Vincentiis, ${ }^{1}$ Giannicola Iannella, ${ }^{1}$ \\ Bruna Cerbelli, ${ }^{2}$ and Giuseppe Magliulo ${ }^{1}$ \\ ${ }^{1}$ Organi di Senso Department, University of “Sapienza”, Viale del Policlinico 151, 00161 Rome, Italy \\ ${ }^{2}$ Pathology Department, University of "Sapienza", Viale Regina Elena 324, 00161 Rome, Italy \\ Correspondence should be addressed to Giuseppe Magliulo; giuseppemagliuloorl@yahoo.com
}

Received 11 June 2015; Revised 18 September 2015; Accepted 20 September 2015

Academic Editor: Chung-Feng Hwang

Copyright (C) 2015 Dario Marcotullio et al. This is an open access article distributed under the Creative Commons Attribution License, which permits unrestricted use, distribution, and reproduction in any medium, provided the original work is properly cited.

Introduction. True malignant mixed tumor, also known as carcinosarcoma, is a rare tumor of the salivary gland composed of both malignant epithelial and malignant mesenchymal elements. Frequently carcinosarcoma arises in the background of a preexisting pleomorphic adenoma; however, if no evidence of benign mixed tumor is present, the lesion is known as carcinosarcoma "de novo." We reported the first case of true malignant mixed tumor of the submandibular gland composed of high grade mucoepidermoid carcinoma associated with osteosarcoma. Case Presentation. A 69-year-old Caucasian male came to our department complaining of the appearance of an asymptomatic left submandibular neoformation progressively increasing in size over 3 months. We opted for surgical treatment. Histological examination confirmed the diagnosis of carcinosarcoma with the coexistence of high grade mucoepidermoid carcinoma and osteosarcoma. Conclusion. To the best of our knowledge, in the true malignant mixed tumor of the submandibular gland, mucoepidermoid carcinoma associated with osteosarcoma has never been previously reported.

\section{Introduction}

True malignant mixed tumor, also known as carcinosarcoma, is an exceedingly rare tumor of the salivary gland composed of both malignant epithelial and malignant mesenchymal elements. Its incidence is comprised between $0.04 \%$ and $0.16 \%$ of all salivary gland tumors with the parotid gland being the most affected site [1-3].

The authors present a recent rare case of 69-year-old man with a malignant mixed tumor of the left submandibular gland consisting in the association of a mucoepidermoid carcinoma (MEC) and an osteosarcoma. To the best of our knowledge, in true malignant mixed tumor of the salivary gland, these microscopic findings have never been previously reported.

Clinical presentation and results of histological and immunohistochemical study are reported.

\section{Case Presentation}

A 69-year-old Caucasian male came to our department complaining of the appearance of an asymptomatic left submandibular neoformation progressively increasing in size over 3 months. Medical, family, and psychosocial history were negative for relevant information; also, no previous surgical treatments were reported.

On clinical examination the mass measured $8 \times 5 \mathrm{~cm}$ arising from level Ib (submandibular region) and extended into levels III and IV. Such mass appeared adherent to the underlying structures with soft texture. No pain or other symptoms were present. Right cervical region, oropharynx, thyroid, and upper respiratory airways showed no involvement.

Ultrasound evaluation of the mass revealed a mixed tissue consistency of cystic and solid areas separated by high flow vascular fibrous septa in level Ib. 


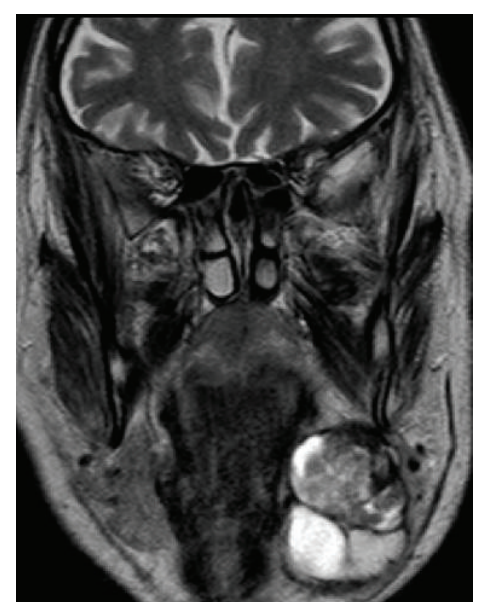

FIgURE 1: Preoperative MRI, coronal T2-W: $5 \times 4 \times 3 \mathrm{~cm}$ mass originating from the left submandibular gland. Two different multilobed neoformations with colliquate areas (black square and black trapezius) delimited by a thick pathological peripheral tissue are clearly visible.

Contrast-enhanced magnetic resonance imaging (MRI) of the head and neck (Figure 1 ) showed a $5 \times 4 \times 3 \mathrm{~cm}$ multilobed neoformation originating from the left submandibular gland. A fluid component and solid areas were inside visible. No distant metastases were detected.

To integrate MRI data, subsequent head and neck computed tomography (CT) was performed. This examination indicated colliquate areas delimited by a thick pathological peripheral tissue within the mass context.

First, fine-needle aspiration cytology specimen was performed to determine the nature of the disease. It showed malignant cells without distinctive features failing to identify the type of primary lesion. A new US-FNAC was subsequently executed; however, also in this case the primary type of lesion was not identified.

We opted for surgical treatment, completely removing the mass by means of ipsilateral neck dissection of Ib, IIa, III, and IV levels.

Histological examination showed a neoplastic proliferation characterized by two cellular components, substantially distinct from each other. No fusion of the different cell types could be seen. The first cellular component consisted of medium size elements with multilobed nucleus and slightly eosinophilic cytoplasm. Such elements appeared arranged in solid cell nests with central necrosis and cribriform areas (Figure 2). Cytokeratin and PAS positivity (Figure 3) were evident. The second cellular component consisted of mesenchymal elements of medium or large size with elongated hyperchromatic and pleomorphic nuclei. Such cellular elements appeared to be arranged around an eosinophilic material attributable to an osteoid matrix (Figure 4). Immunohistochemical study showed vimentin positivity. Tumor showed several cellular atypia, mitoses, areas of necrosis, or bleeding as well as angioinvasion.

Due to the coexistence of two separate cellular patterns, both of malignant nature, a diagnosis of carcinosarcoma,

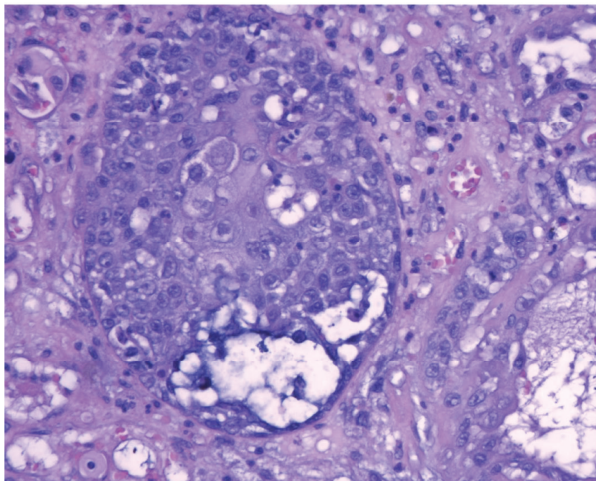

FIGURE 2: Mucoepidermoid carcinoma: glandular-like structure composed of a mixture of squamous and clear cells containing mucin (hematoxylin and eosin, 40x).

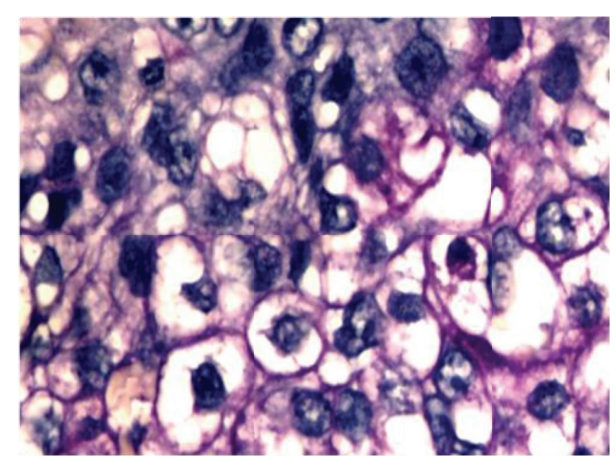

FIGURE 3: Some neoplastic epithelial cells with clear cytoplasm retain PAS positivity after diastase digestion (40x).

obtained by the fusion of high grade mucoepidermoid carcinoma and osteosarcoma, was made.

Postoperatively, the patient underwent intensity-modulated radiation with 66 Gray in 33 cycles.

At six-month follow-up no disease recurrence was revealed.

\section{Discussion}

True malignant mixed tumor (carcinosarcoma) of the salivary gland is an extremely rare tumor in which carcinomatous and sarcomatous components coexist and metastasize together [1-4].

Frequently, carcinosarcoma arises in the background of a preexisting pleomorphic adenoma and, in some cases, tumors were related to a previous history of radiotherapy. However, if none of these conditions is present, the lesion is classified as true malignant mixed tumor or carcinosarcoma "de novo" [3-5]. Table 1 shows the 31 established cases of carcinosarcoma "de novo" actually reported in the English literature. Stephen et al. [6] in 1986 published the largest series of true malignant mixed tumor with 12 cases of carcinosarcoma showed. Malignant epithelial component was ductal carcinoma in all patients, with 1 case of squamous component and 2 with undifferentiated features. About 


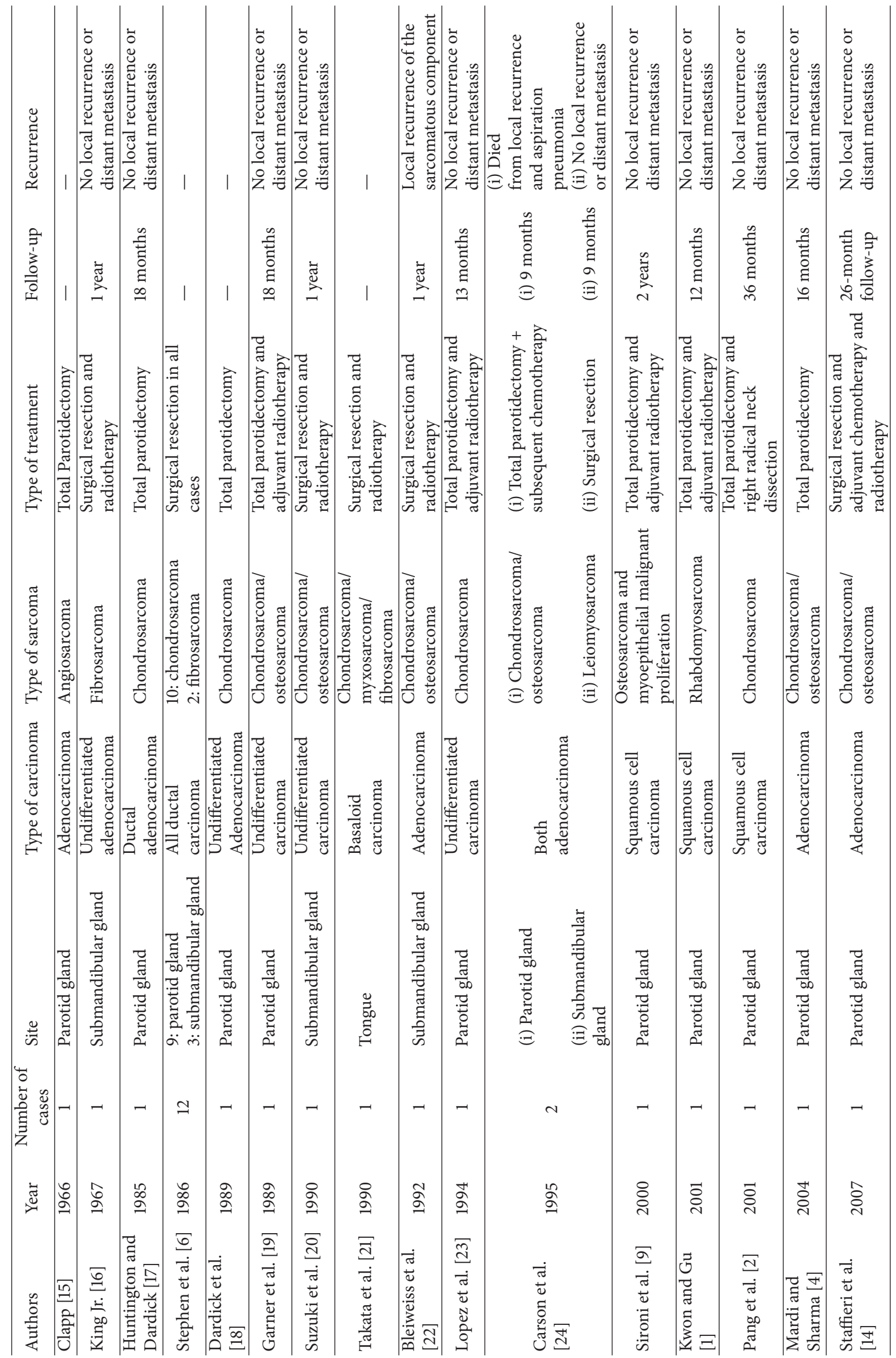




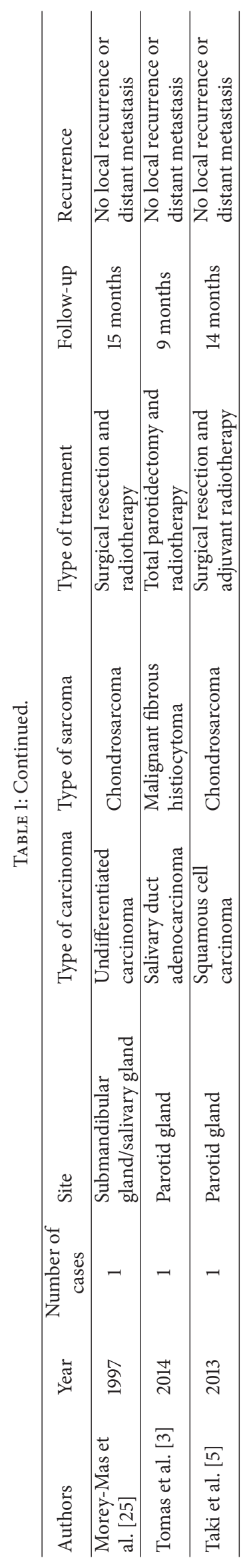




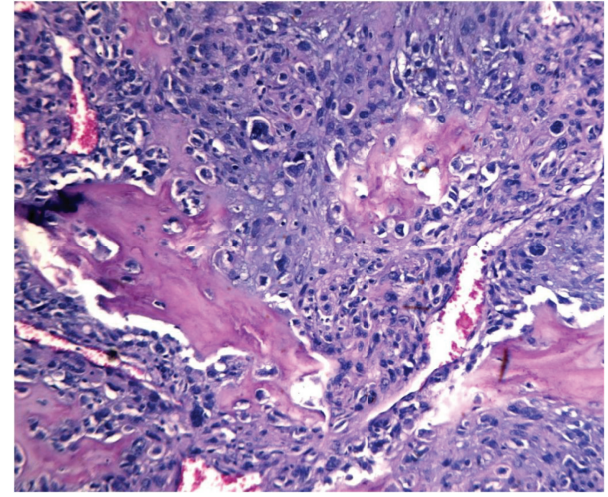

FIGURE 4: Osteosarcoma cells: mesenchymal elements of medium or large size with hyperchromatic, pleomorphic, and multinucleated nuclei arranged around an osteoid matrix (hematoxylin and eosin, 10x).

malignant mesenchymal elements 10 chondrosarcoma and 2 mixed malignant fibrous histiocytoma cases were reported.

The most common malignant epithelial components are squamous cell carcinoma or adenocarcinoma, whereas the malignant mesenchymal component mainly consists of chondrosarcoma, fibrosarcoma, or liposarcoma [1-5].

We reported the first case of true malignant mixed tumor of the salivary gland composed of high grade mucoepidermoid carcinoma and osteosarcoma.

Mucoepidermoid carcinoma is a common salivary tumor derived from ductal epithelium of the salivary gland, which displays a variety of biological behavior patterns. The highgrade variant is more aggressive with a poor prognosis, whereas the low-grade variant usually demonstrates satisfactory survival rates [7]. The diagnosis of MEC includes the identification of three intermixed tumor elements: mucin-producing cells, intermediate and/or clear cells, and squamoid cells $[1,7]$.

Osteosarcoma is the primary malignancy of bone with rare extraosseous head and neck localizations. Typical features of osteosarcoma are the presence of osteoid tissue within the neoformation, with extremely pleomorphic cells included in such osteoid matrix [8].

FNAC has a well-established role in the initial, preoperative diagnosis of salivary gland lesions. It is safe, fast, well tolerated, and minimally invasive; however, it is known to have several deficiencies. On average, FNAC has high specificity (97\%), but the sensitivity is somewhat lower (80\%). Thus, a positive diagnosis by FNAC is quite reliable, but the false-negative rate associated with FNAC (20\%) may be unacceptable [9-12]. In addition, the fine-needle aspiration cytology is not considered effective for the diagnosis of true malignant mixed tumor [10-12]. In our case, FNAC showed malignant cells without distinctive features failing to identify the type of primary lesion.

Core needle biopsy (CNB) is a relatively new technique for the diagnosis of salivary gland masses that offers several potential advantages relative to FNAC $[12,13]$. However, CNB was not performed in our patient.
Histological and immunohistochemical studies are essential both for a correct diagnosis and for distinguishing carcinosarcoma from other tumors. Usually, cytokeratin and epithelial membrane antigens are positive in the carcinomatous element while vimentin positivity is observed in the sarcomatous element [1-5].

No therapeutic protocol has been established for treating this atypical disease, because of limited individual or institutional experience. Treatment may consist of surgery alone or surgery and postoperative radiotherapy $[1,2,14]$. Staffieri et al. [14] compared the carcinosarcoma recurrence data in a group of patients who had undergone surgery versus surgery plus radiotherapy, with lower recurrence rate after the combination of surgery and radiotherapy $(p=0.3)$.

Due to the limited follow-up data reported in the literature, it is very difficult to comment specifically on tumor prognosis. Moreover, the different evolution of the disease could be explained by the histological subtypes observed. Considering 19 cases of de novo parotid carcinosarcoma with available data on follow-up, Staffieri et al. [14] observed that $31.6 \%$ of patients died after a median of 10.1 months from diagnosis. Taki et al. [5] reported a case report of carcinosarcoma consisting of chondrosarcoma and squamous cell carcinoma treated with total parotidectomy and radiation therapy without local or regionally recurrent disease after 14month follow-up. In our case, after 6-month follow-up no disease recurrence was revealed. However, this time is not enough to consider a disease-free survival of our patient.

\section{Conclusions}

Salivary gland carcinosarcoma is a rare and highly aggressive disease with poor prognosis. The current treatment of choice is surgery followed by radiotherapy. However, long-term follow-up with patients who have already undergone treatment is necessary in further elucidating the clinical course of the disease. The association in a true malignant mixed tumor of mucoepidermoid carcinoma and osteosarcoma has never been reported previously, representing therefore a further possibility to be considered.

\section{Conflict of Interests}

The authors declare that they have no conflict of interests.

\section{Acknowledgments}

The authors would like to acknowledge Dr. Ersilia Savastano and Dr. Alessandra Manno of the Sense Organs Department, Sapienza University, Rome, for acquisition and interpretation of data.

\section{References}

[1] M. Y. Kwon and M. Gu, "True malignant mixed tumor (carcinosarcoma) of parotid gland with unusual mesenchymal component: a case report and review of the literature," Archives of Pathology and Laboratory Medicine, vol. 125, no. 6, pp. 812815, 2001. 
[2] P. C. W. Pang, E. W. H. To, W. M. Tsang, and T. L. Liu, "Carcinosarcoma (malignant mixed tumor) of the parotid gland: a case report," Journal of Oral and Maxillofacial Surgery, vol. 59, no. 5, pp. 583-587, 2001.

[3] D. Tomas, D. Vagic, V. Bedekovic, and B. Kruslin, "Carcinosarcoma de novo of the parotid gland with unusual sarcomatous component," Brazilian Journal of Otorhinolaryngology, vol. 80, no. 4, pp. 364-365, 2014.

[4] K. Mardi and J. Sharma, "True malignant mixed tumor (carcinosarcoma) of parotid gland: a case report," Indian Journal of Pathology and Microbiology, vol. 47, no. 1, pp. 64-66, 2004.

[5] N. H. Taki, N. Laver, T. Quinto, and R. O. Wein, "Carcinosarcoma de novo of the parotid gland: case report," Head and Neck, vol. 35, no. 5, pp. E161-E163, 2013.

[6] J. Stephen, J. G. Batsakis, M. A. Luna, U. von der Heyden, and R. M. Byers, "True malignant mixed tumors (carcinosarcoma) of salivary glands," Oral Surgery, Oral Medicine, Oral Pathology, vol. 61, no. 6, pp. 597-602, 1986.

[7] C. H. McHugh, D. B. Roberts, A. K. El-Naggar et al., "Prognostic factors in mucoepidermoid carcinoma of the salivary glands," Cancer, vol. 118, no. 16, pp. 3928-3936, 2012.

[8] R. K. Verma, G. Gupta, A. Bal, and J. Yadav, "Primary giant cell rich osteosarcoma of maxilla: an unusual case report," Journal of Maxillofacial and Oral Surgery, vol. 10, no. 2, pp. 159-162, 2011.

[9] M. Sironi, G. Isimbaldi, R. Claren, C. Delpiano, F. Di Nuovo, and M. Spinelli, "Carcinosarcoma of the parotid gland: cytological, clinicopathological and immunohistochemical study of a case," Pathology Research and Practice, vol. 196, no. 7, pp. 511-517, 2000.

[10] R. L. Schmidt, B. J. Hall, A. R. Wilson, and L. J. Layfield, "A systematic review and meta-analysis of the diagnostic accuracy of fine-needle aspiration cytology for parotid gland lesions," American Journal of Clinical Pathology, vol. 136, no. 1, pp. 4559, 2011.

[11] R. L. Schmidt, J. P. Hunt, B. J. Hall, A. R. Wilson, and L. J. Layfield, "A systematic review and meta-analysis of the diagnostic accuracy of frozen section for parotid gland lesions," American Journal of Clinical Pathology, vol. 136, no. 5, pp. 729738, 2011.

[12] R. L. Schmidt, B. J. Hall, and L. J. Layfield, "A systematic review and meta-analysis of the diagnostic accuracy of ultrasoundguided core needle biopsy for salivary gland lesions," American Journal of Clinical Pathology, vol. 136, no. 4, pp. 516-526, 2011.

[13] R. L. Schmidt, J. D. Jedrzkiewicz, R. J. Allred, S. Matsuoka, and B. L. Witt, "Verification bias in diagnostic accuracy studies for fine- and core needle biopsy of salivary gland lesions in otolaryngology journals: a systematic review and analysis," Head and Neck, vol. 36, pp. 1654-1661, 2014.

[14] C. Staffieri, G. Marioni, S. M. Ferraro, F. Marino, and A. Staffieri, "Carcinosarcoma de novo of the parotid gland," Oral Surgery, Oral Medicine, Oral Pathology, Oral Radiology and Endodontology, vol. 104, no. 2, pp. e35-e40, 2007.

[15] W. A. Clapp, "Histologically benign mixed tumor of the parotid gland with delayed metastases," The Journal of the Maine Medical Association, vol. 57, no. 3, pp. 49-50, 1966.

[16] O. H. King Jr., "Carcinosarcoma of accessory salivary gland. First report of a case," Oral Surgery, Oral Medicine, Oral Pathology, vol. 23, no. 5, pp. 651-659, 1967.

[17] H. W. Huntington and I. Dardick, "Intracranial metastasis from a malignant mixed tumor of parotid salivary gland," Ultrastructural Pathology, vol. 9, no. 3-4, pp. 169-173, 1985.
[18] I. Dardick, J. Hardie, M. J. Thomas, and A. W. P. van Nostrand, "Ultrastructural contributions to the study of morphological differentiation in malignant mixed (pleomorphic) tumors of salivary gland," Head and Neck, vol. 11, no. 1, pp. 5-21, 1989.

[19] S. L. Garner, M. D. Maves, R. A. Robinson, and C. H. Barnes, "Salivary gland carcinosarcoma: true malignant mixed tumor," Annals of Otology, Rhinology \& Laryngology, vol. 98, no. 8, pp. 611-614, 1989.

[20] J. Suzuki, M. Takagi, N. Okada, S. Hatakeyama, and H. Yamamoto, "Carcinosarcoma of the submandibular gland. An autopsy case," Acta Pathologica Japonica, vol. 40, no. 11, pp. 827831, 1990.

[21] T. Takata, H. Nikai, I. Ogawa, and N. Ijuhin, "Ultrastructural and immunohistochemical observations of a true malignant mixed tumor (carcinosarcoma) of the tongue," Journal of Oral Pathology and Medicine, vol. 19, no. 6, pp. 261-265, 1990.

[22] I. J. Bleiweiss, A. G. Huvos, J. Lara, and E. W. Strong, "Carcinosarcoma of the submandibular salivary gland: immunohistochemical findings," Cancer, vol. 69, no. 8, pp. 2031-2035, 1992.

[23] J. I. Lopez, C. Ballestin, M. D. Garcia-Prats, and P. De Agustin, "Carcinosarcoma of the parotid gland: immunohistochemical study of a case," Histopathology, vol. 25, no. 4, pp. 388-390, 1994.

[24] H. J. Carson, D. P. Tojo, J. M. Chow, R. Hammadeh, and W. F. Raslan, "Carcinosarcoma of salivary glands with unusual stromal components. Report of two cases and review of the literature," Oral Surgery, Oral Medicine, Oral Pathology, Oral Radiology and, vol. 79, no. 6, pp. 738-746, 1995.

[25] M. Morey-Mas, J. Caubet-Biayna, C. Gómez-Bellvert, and J. I. Iriarte-Ortabe, "Carcinosarcoma of the submandibular and sublingual salivary glands. A case report and review of the literature.," Acta Stomatologica Belgica, vol. 94, no. 2, pp. 69-73, 1997. 


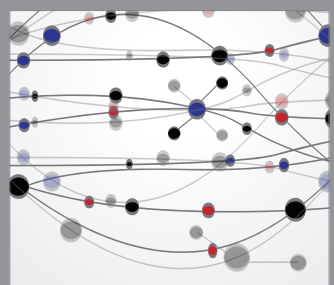

The Scientific World Journal
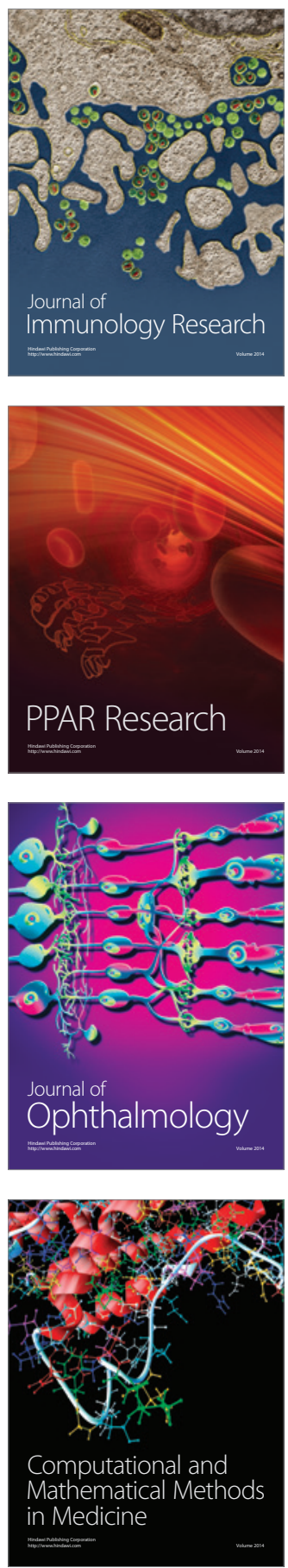

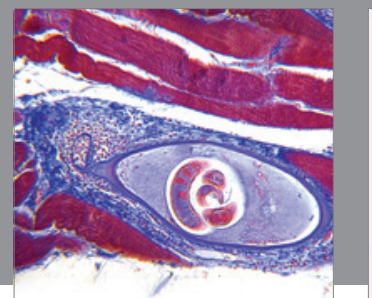

Gastroenterology

Research and Practice
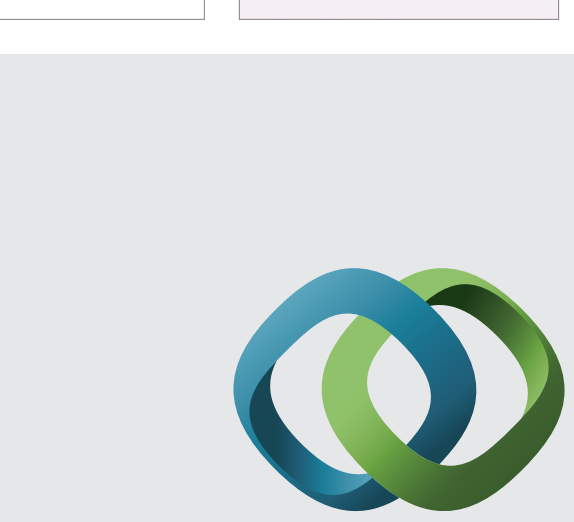

\section{Hindawi}

Submit your manuscripts at

http://www.hindawi.com
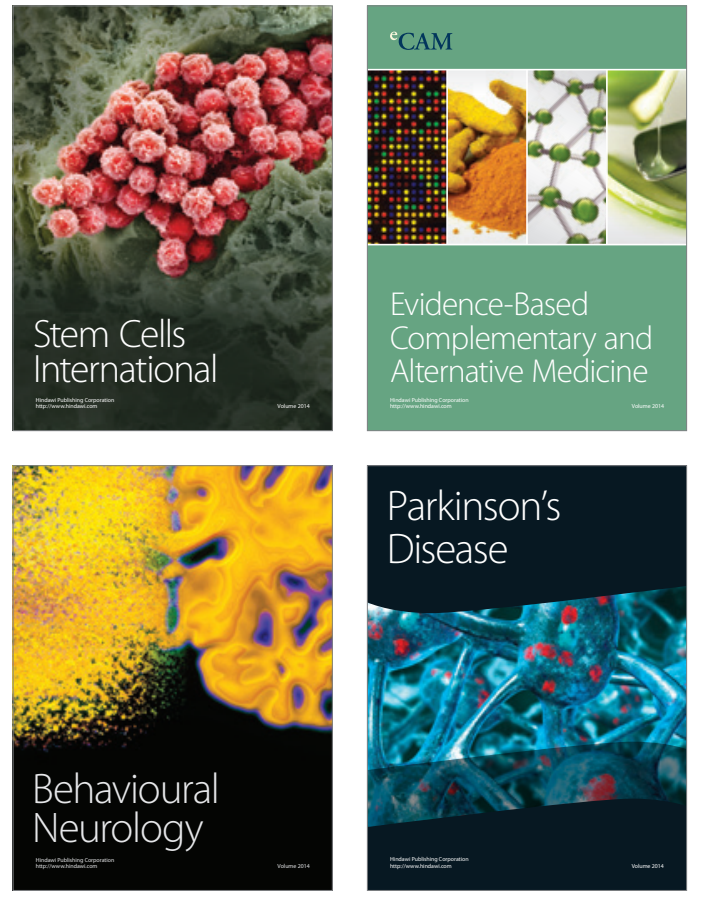
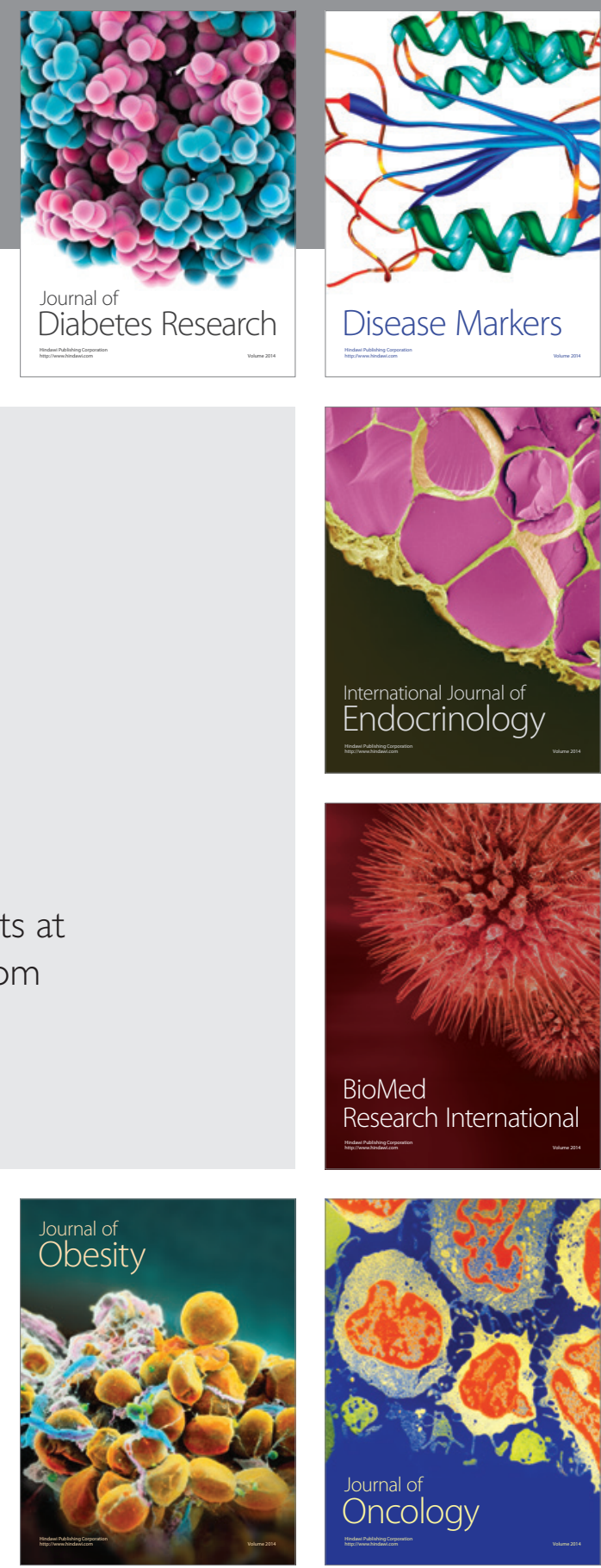

Disease Markers
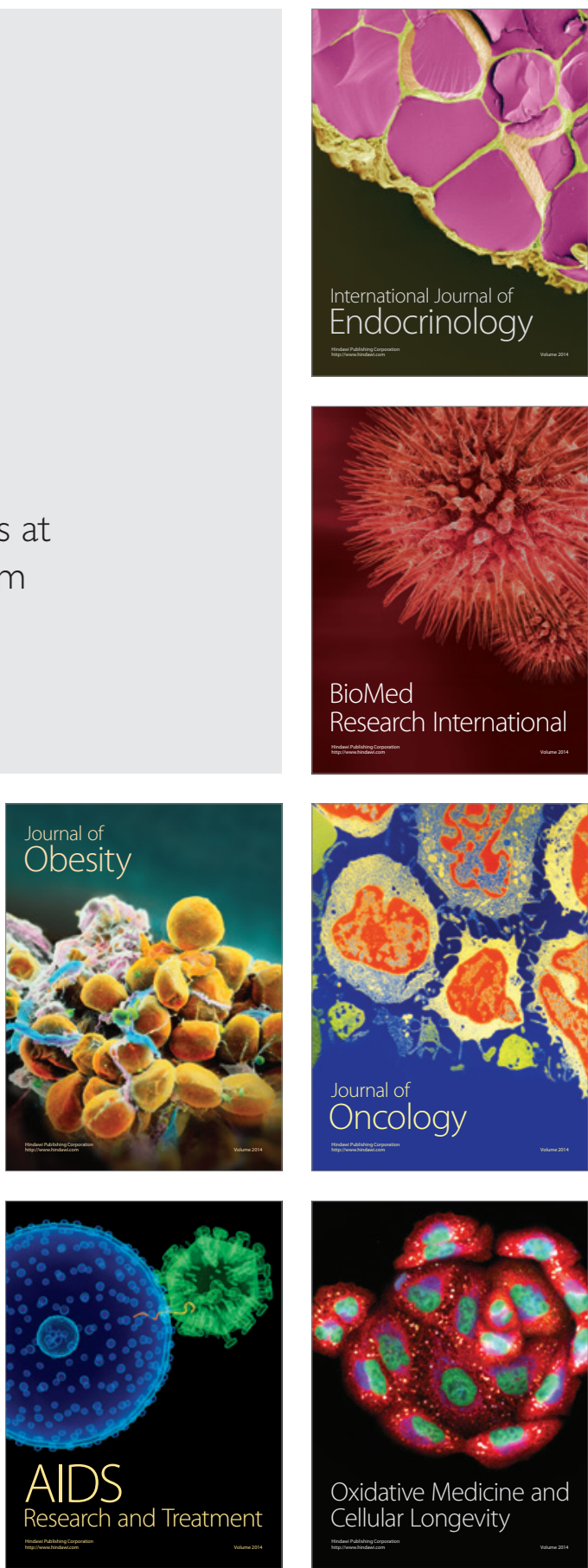